\title{
Production Et Consommation Du Tchapalo À Abidjan : Typologie Des Brasseuses Et Des Consommateurs
}

\author{
Aka Solange \\ Dje Koffi Marcellin
}

Université Nangui Abrogoua, UFR des Sciences et Technologies des Aliments, Laboratoire de Biotechnologie et Microbiologie des Aliments,

Abidjan, Côte d'Ivoire

\author{
Aka Solange \\ Fokou Gilbert \\ Doumbia Mohamed \\ Ahoussi Josiane Marie Stella \\ Bonfoh Bassirou
}

Centre Suisse de Recherches Scientifiques en Côte d'Ivoire (CSRS),

Abidjan, Côte d'Ivoire

\section{Doumbia Mohamed}

\section{Ahoussi Josiane Marie Stella}

Université Félix Houphouët Boigny de Cocody-Abidjan, UFR Sciences de l'Homme et de la Société, Institut d'ethnosociologie, Abidjan, Côte d'Ivoire

doi: 10.19044/esj.2017.v13n1p148 ～$\quad$ URL:http://dx.doi.org/10.19044/esj.2017.v13n1p148

\begin{abstract}
The tchapalo, traditional Ivorian beer, formerly produced in northern Côte d'Ivoire, has overflowed the geographical limits of its production. Its consumption is becoming more momentum in Abidjan. For a better knowledge of actors and a valorization of this beverage, this work was conducted through a survey of 385 brewers and 77 tchapalo consumers selected in 3 areas of Abidjan. Results showed that tchapalo is produced exclusively by Burkinabe women $(45.5 \%)$ and the Ivorian women came from northern part of Côte d'Ivoire (53.2\%), mainly women of the Lobi ethnic group (90.2\%). Tchapalo production was a significant source of income for these women. The biggest consumers of tchapalo came from the Akan ethnic group which represented $52.5 \%$ of respondents. Because of its financial accessibility (300 CFA francs per liter), tchapalo is consumed by all socio-economic and professional groups, particularly foremen (41\%) and laborers (31.7\%). Consumers who found therapeutic principles to the tchapalo represented
\end{abstract}


$54.3 \%$ of respondents. Furthermore, over $95 \%$ of respondents preferred to consume into tchapalo production area to meet friends and also to maintain fraternity links with brewers. However, $25.7 \%$ of consumers chose to consume in a specific tchapalo production area for the taste of the beverage and $25.5 \%$ due to welcome that their reserves the producer. Favorite consumption moments were afternoons and evenings.

Keywords: Tchapalo, tchapalo production area, brewers, consumers, Abidjan

\section{Résumé}

Le tchapalo, bière traditionnelle ivoirienne, jadis produite dans le Nord de la Côte d'Ivoire, a débordé les cadres géographiques de sa production. Sa consommation prend de plus en plus de l'ampleur à Abidjan. Pour une meilleure connaissance des acteurs et une valorisation de cette boisson, le présent travail a été réalisé à travers une enquête auprès de 77 productrices et 385 consommateurs de tchapalo sélectionnés dans 3 communes d'Abidjan. Les résultats ont montré que le tchapalo est produit exclusivement par les femmes burkinabés $(45,5 \%)$ et les femmes ivoiriennes du nord de la Côte d'Ivoire (53,2\%), principalement les femmes de l'ethnie Lobi (90,2\%). La production du tchapalo était une source de revenue non négligeable pour ces femmes. Les plus grands consommateurs de tchapalo étaient issus du groupe ethnique Akan et représentaient 52,5\% des enquêtés. Du fait de son accessibilité financière (300 franc cfa le litre), le tchapalo est consommé par toutes les couches socio-économique et professionnelles, singulièrement les agents de maîtrise (41\%) et les ouvriers (31,7\%). Les consommateurs qui trouvaient des principes thérapeutiques au tchapalo représentaient $54,3 \%$ des enquêtés. Par ailleurs, plus de 95\% des personnes interrogées préféraient consommer au tchapalodrôme pour se retrouver entre amis et entretenir également des liens de fraternités avec les brasseuses. Cependant, 25,7\% des consommateurs choisissaient de consommer dans un tchapalodrôme précis pour le goût de la boisson et 25,5\% à cause du bon accueil que leur réserve la productrice. Les moments favoris de consommation étaient les après-midis et les soirées.

Mots-clés : Tchapalo, tchapalodrôme, brasseuses, consommateurs, Abidjan

\section{Introduction}

En Afrique et particulièrement en Côte d'Ivoire, les céréales sont souvent utilisées pour produire des boissons telles que le moût sucré et le tchapalo (Kouame et al., 2015). Le tchapalo est obtenu à partir de la fermentation alcoolique de moût sucré provenant de malt de sorgho. Il peut 
être aussi produit à partir du malt de maïs ou de mil. Le tchapalo est une bière traditionnelle trouble, peu alcoolisée, contenant des matières en suspension et de la levure. Cette boisson est aussi caractérisée par une courte durée de conservation ( 3 jours) et une qualité qui varie d'une production à une autre (Kouame et al., 2015).

Le tchapalo est très prisé par la population ivoirienne (Yao et al., 1995 ; Djè et al., 2008 ; N'Guessan, 2009). Il est au cœur des cérémonies et réjouissances populaires (mariage, baptême, initiation, funérailles). A ce titre il joue un rôle socio-culturel et économique très important. Le tchapalo est également consommé pendant les travaux champêtres et donné à l'étranger pour se rafraîchir et lui souhaiter la bienvenue (Aka et al., 2010 ; Oyewole and Isah, 2012). Cette boisson possède des valeurs nutritives qui contribuent à améliorer le régime alimentaire des populations consommatrices. De plus, des vertus thérapeutiques lui sont attribuées du fait de ses propriétés laxative, antipaludéenne et anti-hémorroïdaire (Enou, 1997 ; Amané et al., 2005 ; Aka et al., 2010). Son coût relativement bas en fait également un produit à la portée de toutes les bourses (Yao et al., 1995 ; Djè et al., 2008 ; N'Guessan, 2009).

Dans son processus technologique empirique, interviennent un maltage, une mouture, un empattage, une pré-cuisson, un aigrissement ou fermentation spontanée, une filtration, une cuisson, un refroidissement, une inoculation et une fermentation alcoolique. La production du tchapalo était jadis localisée dans le nord de la Côte d'Ivoire et destinée à la consommation journalière familiale (N'Guessan, 2009 ; Amane et al., 2012). Mais de nos jours, cette production est passée du cadre familial à une production commerciale. Elle est essentiellement assurée par les femmes appartenant aux groupes ethniques du Nord de la Côte d'Ivoire (Nord-Centre et Nord-Est). Celles-ci en ont fait, une véritable activité économique génératrice de revenu dans toutes les villes du pays et particulièrement à Abidjan, la capitale économique où l'on dénombre plus d'une centaine de tchapalodrôme (lieux de production et de commercialisation du tchapalo) (Yao et al., 1995 ; Enou, 1997 ; Aka et al., 2008). Il s'est ainsi développé une filière bière de sorgho qui fait vivre plusieurs familles en Côte d'Ivoire et même dans la sous-région (N'Guessan, 2009 ; Pale et al., 2011). Toutefois, à notre connaissance, il n'existe aucune donnée scientifique en Côte d'Ivoire, sur les acteurs impliqués dans la production et la consommation du tchapalo. Cette quasi-absence de données scientifiques montre l'intérêt de cette étude.

L'objectif de ce travail est de présenter la typologie des brasseuses et consommateurs du tchapalo à Abidjan pour une meilleure connaissance des acteurs de la filière bière de sorgho en Côte d'Ivoire. 


\section{Méthodologie}

A l'issu d'une préenquête, trois communes d'Abidjan ont été sélectionnées pour cette étude parce qu'elles renferment les plus grands nombres de tchapalodrômes (données d'enquête) à savoir Abobo, Cocody et Yopougon (Figure 1). Le sondage stratifié à trois niveaux a été utilisé comme méthode d'échantillonnage pour sélectionner 77 productrices et la technique d'échantillon accidentel a été utilisée pour interroger 385 consommateurs de tchapalo dans ces trois communes d'Abidjan. L'enquête s'est déroulée du 05 Juin au 18 Décembre 2014. Les questionnaires ont porté entre autres sur le statut sociodémographique (identité, genre, âge, ethnie, région d'origine) des productrices et des consommateurs ainsi que leur situation socio-économique, les caractéristiques spécifiques du tchapalo perçues par les consommateurs, les habitudes de consommation, les raisons de consommation du tchapalo, les fréquences et les moments de consommation.

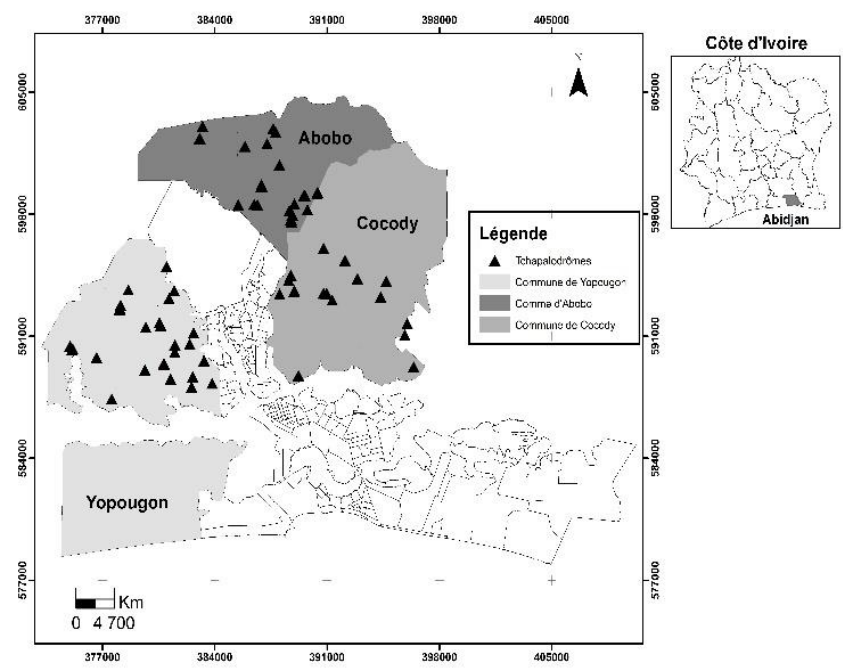

Figure 1: Carte de distribution spatiale des tchapalodrômes enquêtés dans les trois communes d'Abidjan.

\section{Analyse des données issues de l'enquête}

Les différentes informations collectées sur les fiches d'enquête ont été saisies avec le logiciel Epi-data 3.0 puis transférées dans le logiciel SPSS version 20.0 pour constituer la base de données. Les pourcentages ont été calculés pour les variables quantitatives ainsi que les moyennes et les écarttypes. 


\section{Résultats et discussion}

Typologie des brasseuses de tchapalo à Abidjan

- Profil sociodémographique des brasseuses

L'enquête réalisée auprès des brasseuses de tchapalo a montré que cette boisson était uniquement produite par les femmes (Tableau 1). Ce constat s'expliquerait par le fait qu'en Afrique, tout ce qui est lié à la cuisine ou à l'alimentation est dévolu à la femme. La majorité de ces productrices faisait partie de la population active (15 - 45 ans) de la Côte d'Ivoire. Cette population représentait $75,4 \%$ des productrices enquêtées. Par ailleurs, plus de la moitié des brasseuses interrogées $(53,2 \%)$ était de nationalité Ivoirienne ; 45,5\% de nationalité Burkinabé et $1,3 \%$ de nationalité Togolaise. L'importante proportion de brasseuses Burkinabés dans la population étrangère s'expliquerait par le fait que l'histoire du tchapalo est liée à un certain nombre de pays dont le Burkina Faso, pays voisin de la Côte d'Ivoire. Dans ce pays, le tchapalo appelé dolo est la boisson alcoolisée la plus consommée (Abdoullatif et al., 2013). En outre, le Burkina Faso est fortement représenté en Côte d'Ivoire à travers ses ressortissants (Zongo, 2003). Le processus de migration a non seulement permis aux Burkinabés de s'installer à Abidjan, mais également de renforcer leur présence dans la production du dolo.

Parmi les 53,2\% de brasseuses ivoiriennes, 90,2\% étaient de l'ethnie Lobi et 4,9\% de l'ethnie Sénoufo. La forte présence des Lobis s'expliquerait par le fait que la production du tchapalo fait partie intégrante des habitudes culinaires de ce peuple. Ces résultats confirment donc l'origine du tchapalo en Côte d'Ivoire. En effet, plusieurs auteurs affirment que le tchapalo provient du Nord de la Côte d'Ivoire (Aka et al., 2008 ; N'Guessan, 2009 ; Amane et al., 2012). Au niveau de l'éducation des productrices, $80,5 \%$ des productrices n'avaient aucun niveau d'étude. Seulement, $10,4 \%$ et 7,8\% d'entre elles avaient respectivement le niveau primaire et le niveau secondaire. Les données révèlent aussi que 55,9\% des productrices enquêtées étaient en union (mariées et les personnes en concubinage).

\section{- Profil socio-économique des brasseuses}

Le profil socio-économique des productrices de tchapalo à Abidjan est présenté dans le tableau 1. Les productrices résidaient dans les communes enquêtées et plusieurs d'entre elles vivaient soit dans des « cours communes » (61\%), soit dans des baraques en bois communément appelé sicobois $(24,7 \%)$. Les cours communes sont des cours où habitent plusieurs familles d'origines diverses qui ont un revenu plus ou moins modeste voire faible. Environ 44,1\% des brasseuses enquêtées étaient propriétaires de tchapalodrôme tandis que $42,9 \%$ louaient le tchapalodrôme et les $13 \%$ restantes travaillaient pour le compte d'une tierce personne qui est généralement la responsable du tchapalodrôme. 
Pour une utilisation de $100 \mathrm{~kg}$ de sorgho, 90,9\% des productrices ont affirmé gagner entre 5000 francs CFA et 10000 francs CFA ; tandis que 5,2\% d'entre elles gagnaient autour de 15000 francs CFA pour la même utilisation de quantité de sorgho. La vente du tchapalo est donc une activité qui constitue une source de revenu non négligeable pour les productrices (Maoura et al., 2006 ; Pale et al., 2011 ; Abdoul-latif et al., 2012 ; Yaméogo et al., 2013). Elle permet aux brasseuses de se prendre en charge ainsi que leurs familles.

Par ailleurs, le sac de $100 \mathrm{~kg}$ de sorgho était utilisé pour 3 productions selon $90,9 \%$ des productrices enquêtées ou pour 2 productions selon $9,1 \%$ ? des brasseuses. Les $90,9 \%$ utilisaient 200 litres d'eau pour chaque production et obtenaient entre 70 et 85 litres de tchapalo tandis que les autres utilisaient environ 380 litres d'eau pour produire 120 à 135 litres de tchapalo.

Tableau 1: Profils sociodémographiques et socio-économiques des brasseuses de tchapalo à

\begin{tabular}{|c|c|c|c|c|c|c|c|}
\hline \multirow{3}{*}{$\begin{array}{l}\text { Observat } \\
\text { ions }\end{array}$} & \multirow{3}{*}{$\begin{array}{c}\text { Caractéristiques } \\
\text { socio- } \\
\text { démographique } \\
\mathrm{s}\end{array}$} & \multicolumn{4}{|c|}{ Abidjan } & \multicolumn{2}{|c|}{ 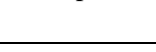 } \\
\hline & & \multicolumn{2}{|c|}{$\begin{array}{l}\text { Brasseuse } \\
\mathrm{s}(\mathrm{n}=77)\end{array}$} & \multirow[t]{2}{*}{ Observations } & \multirow{2}{*}{$\begin{array}{l}\text { Caractéristiqu } \\
\text { es socio- } \\
\text { économiques }\end{array}$} & \multicolumn{2}{|c|}{$\begin{array}{c}\text { Brasseuse } \\
\mathrm{s}(\mathrm{n}=77)\end{array}$} \\
\hline & & $\begin{array}{l}\text { Eff } \\
\text { ect } \\
\text { ifs }\end{array}$ & $\begin{array}{l}\text { Pour } \\
\text { cent } \\
\text { age } \\
(\%) \\
\end{array}$ & & & $\begin{array}{l}\text { Eff } \\
\text { ect } \\
\text { ifs }\end{array}$ & $\begin{array}{l}\text { Pour } \\
\text { cent } \\
\text { age } \\
(\%)\end{array}$ \\
\hline Genre & Féminin & 77 & 100 & Type d'habitat & Appartement & 4 & 5,2 \\
\hline \multirow[t]{5}{*}{$\begin{array}{l}\text { Age } \\
\text { (ans) }\end{array}$} & {$[15-25[$} & 6 & 7,8 & & $\begin{array}{l}\text { Cours } \\
\text { commune }\end{array}$ & 47 & 61,0 \\
\hline & {$[25-35[$} & 23 & 29,9 & & Sicobois & 19 & 24,7 \\
\hline & [35-45[ & 29 & 37,7 & & Villa & 3 & 3,9 \\
\hline & {$[45-55[$} & 15 & 19,5 & & Autre & 4 & 5,2 \\
\hline & [55-plus [ & 4 & 5,2 & Nombre de & {$[0-6[$} & 53 & 68,8 \\
\hline \multirow{3}{*}{$\begin{array}{l}\text { Nationali } \\
\text { té }\end{array}$} & Ivoirienne & 41 & 53,2 & personnes à charge & {$[6-11[$} & 22 & 28,6 \\
\hline & Burkinabé & 35 & 45,5 & & [11-plus[ & 2 & 2,6 \\
\hline & Togolaise & 1 & 1,3 & Statut du & Propriétaire & 34 & 44,1 \\
\hline \multirow{3}{*}{$\begin{array}{l}\text { Groupe } \\
\text { ethnique }\end{array}$} & Akan & 1 & 2,4 & tchapalodrôme & Locataire & 33 & 42,9 \\
\hline & Gur & 39 & 95,2 & & Autre & 10 & 13,0 \\
\hline & Krou & 1 & 2,4 & $\begin{array}{l}\text { Gains pour } 100 \mathrm{~kg} \text { de } \\
\text { production de }\end{array}$ & $\begin{array}{c}{\left[\begin{array}{c}5 \\
0\end{array} 000-10000\right.} \\
{[}\end{array}$ & 70 & 90,9 \\
\hline \multirow[t]{4}{*}{ Ethnie } & Lobi & 37 & 90,2 & sorgho (FCFA) & $\begin{array}{c}{[10000-15} \\
000[\end{array}$ & 4 & 5,2 \\
\hline & Sénoufo & 2 & 4,9 & & $\geq 15000$ & 1 & 1,3 \\
\hline & Bété & 1 & 2,4 & & $\begin{array}{l}\text { Non } \\
\text { répondant }\end{array}$ & 2 & 2,6 \\
\hline & Abbey & 1 & 2,4 & Nombre de & 3 fois & 70 & 90,9 \\
\hline \multirow{4}{*}{$\begin{array}{l}\text { Niveau } \\
\text { d'instruct } \\
\text { ion }\end{array}$} & Aucun & 62 & 80,5 & $\begin{array}{l}\text { production pour } \\
100 \mathrm{~kg} \text { de sorgho }\end{array}$ & 2 fois & 7 & 9,1 \\
\hline & Ecole coranique & 1 & 1,3 & Volume d'eau & 2001 & 70 & 90,9 \\
\hline & Primaire & 8 & 10,4 & $\begin{array}{l}\text { utilisée par } \\
\text { production }\end{array}$ & 3801 & 7 & 9,1 \\
\hline & Secondaire & 6 & 7,8 & & $70-851$ & 70 & 90,9 \\
\hline
\end{tabular}




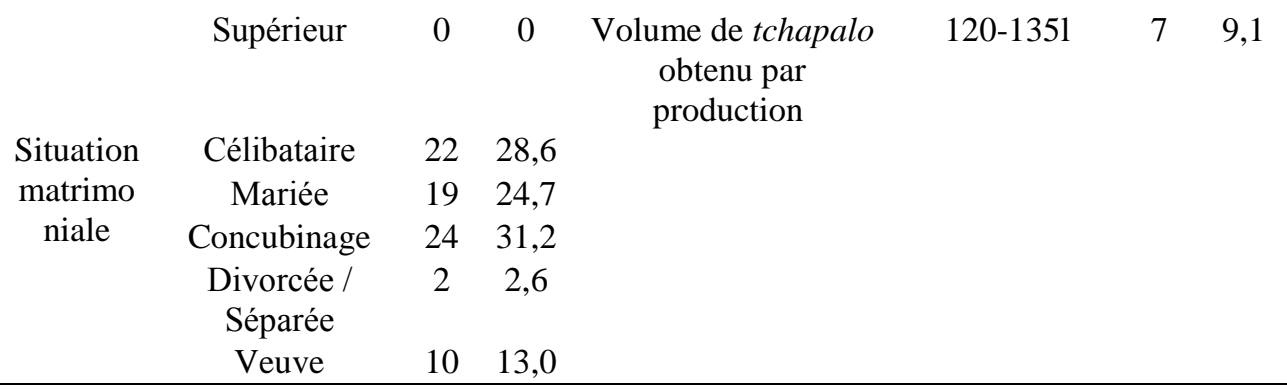

\section{Typologie des consommateurs de tchapalo à Abidjan}

\section{- Profil sociodémographique des consommateurs}

L'enquête sur la consommation de tchapalo a montré que les hommes étaient de grands consommateurs de tchapalo (96,9\% des consommateurs de tchapalo). La consommation de bières locales par les hommes a été également montré dans les études de Kayodé (2006) sur le tchoukoutou, une bière traditionnelle produite au Benin et dans celles de Pale et al. (2011) et Abdoullatif et al. (2013) sur le dolo au Burkina Faso. Les consommateurs dont l'âge était compris entre 25-35 ans et 35-45 ans constituaient la majorité des personnes enquêtées et représentaient respectivement $39,7 \%$ et $34,6 \%$ des consommateurs (Tableau 2). Après ces deux catégories d'individus, venaient ceux dont l'âge était compris entre 45 et 55 ans (13,5\%). La forte représentation de consommateurs âgés de 25 à 45 ans reflète l'image de la population ivoirienne qui est en majorité jeune (INS, 2015).

$\mathrm{Au}$ niveau de la nationalité, les Ivoiriens représentaient $72,7 \%$ des consommateurs enquêtés contre $23,6 \%$ de Burkinabé et seulement $3,7 \%$ de consommateurs venant d'autres pays d'Afrique. Parmi les Ivoiriens, 52,5\% étaient du groupe ethnique Akan. Ensuite venaient les Gur (22,5\%) puis les Krou $(10,5 \%)$ et les Mandé du sud (10\%). Les Mandé du nord étaient faiblement représentés $(2,5 \%)$. Les données montrent la diversité culturelle de la Côte d'Ivoire en lien avec la consommation du tchapalo. En effet, la population ivoirienne est constituée d'une soixantaine d'ethnies regroupées en cinq grands groupes ethniques, à savoir : les Akan, les Krou, les Mandé du Sud, les Mandé du Nord et les Gur (Akoa et al., 2012 ; INS/ICF, 2012). La forte présence des Akan montre ainsi que cette bière n'est plus une boisson exclusivement réservée aux nordistes. Ces résultats sont en conformité avec ceux de Enou (1997) qui a montré que les Akans sont en majorité des consommateurs de tchapalo à Abidjan. Aussi, la consommation du tchapalo à Abidjan serait-elle liée à d'autres facteurs qu'une simple question d'habitudes culturelles, propre à tel ou tel groupe ethnique.

En outre, $37,9 \%$ des consommateurs, avaient le niveau secondaire et $23,9 \%$ avaient le niveau primaire, $18,7 \%$ le niveau supérieur et seulement $17,1 \%$ n'ont jamais été scolarisés. Ces résultats montrent que la consommation 
du tchapalo n'est pas le seul fait des personnes sans niveau d'instruction ou de faible niveau d'instruction.

Plusieurs consommateurs vivaient en couple (marié ou en concubinage). Ceux-ci représentaient $54,2 \%$ de la population enquêtée. La proportion des célibataires n'était pas non plus négligeable $(43,4 \%)$. Ainsi, quel que soit la situation matrimoniale, toutes les catégories sociales consomment le tchapalo.

\section{- Profils socio-économiques et capital économique des consommateurs}

Les consommateurs interrogés appartenaient à différentes catégories socio-professionnelles (Tableau 2). Ainsi, $41 \%$ des consommateurs appartenaient à la catégorie des employés ou agents de maîtrise. La population des ouvriers (employés des usines, vigiles, mécaniciens) n’était pas non plus négligeable. Elle représentait $31,7 \%$ des personnes interrogées. Les commerçants $(4,7 \%)$, les cadres $(2,9 \%)$, les exploitants agricoles $(1,6 \%)$ bien qu'ils représentassent une proportion infime des enquêtés, faisaient partie de la population active. Toutes les catégories socio-professionnelles seraient concernées par la consommation du tchapalo. Ces résultats rejoignent ceux de Akoma et al. (2013) qui ont aussi mentionné que toutes les classes sociales consomment le Kunun-Zaki, une boisson à base de céréales produite dans le Nord du Nigeria.

Outre les catégories socio-professionnelles, 55,8\% des consommateurs interrogés habitaient dans les cours communes et 13,3\% dans des sicobois. Seulement $12,2 \%$ et $10,4 \%$ des consommateurs habitaient respectivement dans un appartement et dans une villa. Les consommateurs de tchapalo qui habitaient dans les cours communes et les sicobois étaient des personnes à faible revenu. Le fait que le tchapalo soit consommé en grande majorité par ces individus montre qu'il est économiquement accessible. Ces résultats sont en conformité avec ceux de Djanan et al. (2002) et de Magrin et M'Bayoudel (2005) qui ont mentionné qu'au Tchad, les consommateurs sont majoritairement jeunes et sont des personnes à faible revenu. En effet la répartition des consommateurs par revenu mensuel a montré que ceux qui avaient un revenu mensuel inférieur ou égal à 100.000 F CFA étaient les plus nombreux $(54,8 \%)$. Néanmoins, le tchapalo est également consommé par des individus de classe sociale aisée en l'occurrence des cadres supérieurs (2,9\%). Le tchapalo est donc une bière accessible. Le litre de tchapalo coûte 300 franc CFA contre 800 franc pour la bière moderne « bock $^{1}$ ». C'est l'une des bières les moins coûteuses du marché ivoirien.

\footnotetext{
${ }^{1}$ Bock est le nom de la bière produite par la Société de limonadérie et de brasserie d'Afrique (SOLIBRA) en Côte d'Ivoire. Cette bière est la plus consommée par les ivoirien et la plus accessible ; 600 francs CFA pour la bouteille de $66 \mathrm{cl}$ et 800 francs CFA pour la bouteille de $100 \mathrm{cl}$ (1 litre).
} 
Le tchapalo est accessible financièrement au point où les consommateurs dépensant entre 300 francs et 600 francs au tchapalodrôme étaient les plus nombreux. Ils représentaient 39,7\% des consommateurs enquêtés (Tableau 2). Ceci confirme l'accessibilité financière de cette boisson. Quel que soit leur catégorie socioprofessionnelle, les dépenses effectuées par les consommateurs n'excédaient pas 1800 FCFA.

\section{Appréciation de la qualité du tchapalo par les consommateurs}

L'élément principal qui permet aux consommateurs de savoir si le tchapalo est bon à consommer était le goût (Tableau 2). Pour 82,6\% des consommateurs enquêtés, le goût était le critère essentiel pour apprécier la qualité du tchapalo contre 4,9\% des consommateurs qui utilisaient 1'odeur comme élément d'appréciation de la qualité du tchapalo. Par contre, pour différencier le tchapalo d'un jour à celui de plusieurs jours, plus de la moitié des consommateurs, quel que soit le nombre d'année de consommation, considéraient le degré d'alcool. Ces résultats rejoignent ceux de Pale et al. (2010) qui ont aussi montré que le critère le plus important utilisé par les consommateurs au Burkina Faso pour évaluer la qualité du dolo est le goût suivi de la teneur en alcool. Plus de $80 \%$ des consommateurs interrogés ont affirmé que la durée de conservation du tchapalo se situe entre deux jours et une semaine contre $37,1 \%$ qui ont estimé cette durée à exactement 3 jours.

Tableau 2: Profils sociodémographiques et socioéconomique des consommateurs de tchapalo à Abidjan

\begin{tabular}{|c|c|c|c|c|c|c|c|}
\hline \multirow[t]{2}{*}{$\begin{array}{l}\text { Observat } \\
\text { ions }\end{array}$} & \multirow[t]{2}{*}{$\begin{array}{l}\text { Caractéristiques } \\
\text { socio- } \\
\text { démographiques }\end{array}$} & \multicolumn{2}{|c|}{$\begin{array}{c}\text { Consommat } \\
\text { eurs } \\
(\mathrm{n}=385) \\
\end{array}$} & \multirow[t]{2}{*}{ Observations } & \multirow[t]{2}{*}{$\begin{array}{c}\text { Caractéristiques } \\
\text { socio- } \\
\text { économiques }\end{array}$} & \multicolumn{2}{|c|}{$\begin{array}{c}\text { Consommat } \\
\text { eurs } \\
(\mathrm{n}=385)\end{array}$} \\
\hline & & $\begin{array}{c}\text { Eff } \\
\text { ecti } \\
\text { fs } \\
\end{array}$ & $\begin{array}{l}\text { Pourc } \\
\text { entag } \\
\text { e }(\%) \\
\end{array}$ & & & $\begin{array}{c}\text { Eff } \\
\text { ecti } \\
\text { fs }\end{array}$ & $\begin{array}{l}\text { Pourc } \\
\text { entag } \\
\text { e }(\%) \\
\end{array}$ \\
\hline \multirow[t]{2}{*}{ Genre } & Féminin & 12 & 3,1 & \multirow{4}{*}{$\begin{array}{c}\text { Situation } \\
\text { matrimoniale }\end{array}$} & Célibataire & 167 & 43,4 \\
\hline & Masculin & 373 & 96,9 & & Marié & 86 & 22,3 \\
\hline Age & {$[15-$} & 14 & 3,6 & & Concubinage & 123 & 31,9 \\
\hline \multirow[t]{4}{*}{ (ans) } & {$[25-35[$} & 153 & 39,7 & & $\begin{array}{l}\text { Divorcé / } \\
\text { Séparé }\end{array}$ & 3 & 0,8 \\
\hline & {$[35-45[$} & 133 & 34,6 & & Veuf/Veuve & 6 & 1,6 \\
\hline & [45-55[ & 52 & 13,5 & Type & Appartement & 47 & 12,2 \\
\hline & [55-plus[ & 33 & 8,6 & d'habitat & Cours commune & 215 & 55,8 \\
\hline \multirow{4}{*}{$\begin{array}{l}\text { National } \\
\text { ité }\end{array}$} & Ivoirienne & 280 & 72,7 & & Sicobois & 51 & 13,3 \\
\hline & Burkinabé & 91 & 23,6 & & Villa & 40 & 10,4 \\
\hline & Guinéen & 2 & 0,5 & & Autre & 28 & 7,3 \\
\hline & Autre Afrique & 12 & 3,2 & & Non répondant & 4 & 1 \\
\hline \multirow{4}{*}{$\begin{array}{l}\text { Groupe } \\
\text { ethnique }\end{array}$} & Akan & 202 & 52,5 & Nombre de & {$[0-6[$} & 293 & 76,1 \\
\hline & $\mathrm{G}$ & 87 & 22,5 & personnes à & [6-11[ & 56 & 14,5 \\
\hline & Krou & 40 & 10,5 & charge & [11-plus[ & 8 & 2,1 \\
\hline & Mandé du nord & 10 & 2,5 & & Non répondant & 28 & 7,3 \\
\hline
\end{tabular}




\begin{tabular}{cccc} 
& Mandé du sud & 38 & 10 \\
Niveau & Naturalisé & 8 & 2 \\
d'instruct & Aucun & 66 & 17,2 \\
ion & Ecole coranique & 5 & 1,3 \\
& Primaire & 92 & 23,9 \\
& Secondaire & 146 & 37,9 \\
& Supérieur & 72 & 18,7 \\
& Non répondant & 4 & 1 \\
\hline
\end{tabular}

Tableau 2 : Profils sociodémographiques et socioéconomique des consommateurs de tchapalo à Abidjan (suite)

\begin{tabular}{|c|c|c|c|c|c|c|c|}
\hline \multirow[t]{2}{*}{$\begin{array}{l}\text { Observati } \\
\text { ons }\end{array}$} & \multirow[t]{2}{*}{$\begin{array}{l}\text { Caractéristiques } \\
\text { socio- } \\
\text { démographiques }\end{array}$} & \multicolumn{2}{|c|}{$\begin{array}{c}\text { Consomma } \\
\text { teurs } \\
(\mathrm{n}=385) \\
\end{array}$} & \multirow[t]{2}{*}{ Observations } & \multirow[t]{2}{*}{$\begin{array}{c}\text { Caractéristique } \\
\text { s socio- } \\
\text { économiques }\end{array}$} & \multicolumn{2}{|c|}{$\begin{array}{c}\text { Consomma } \\
\text { teurs } \\
(\mathrm{n}=385) \\
\end{array}$} \\
\hline & & $\begin{array}{c}\text { Eff } \\
\text { ecti } \\
\text { fs }\end{array}$ & $\begin{array}{l}\text { Pour } \\
\text { centa } \\
\text { ge } \\
(\%)\end{array}$ & & & $\begin{array}{l}\text { Eff } \\
\text { ecti } \\
\text { fs }\end{array}$ & $\begin{array}{l}\text { Pourc } \\
\text { entag } \\
\text { e }(\%)\end{array}$ \\
\hline \multirow{9}{*}{$\begin{array}{l}\text { Catégorie } \\
\text { socio- } \\
\text { profession } \\
\text { nelle }\end{array}$} & Cadres & 11 & 2,9 & Dépenses & $<300 \mathrm{fcfa}$ & $\overline{56}$ & 14,6 \\
\hline & Commerçant & 18 & 4,7 & $\begin{array}{l}\text { effectuées par } \\
\text { consommation }\end{array}$ & $300-600 \mathrm{fcfa}$ & $\begin{array}{c}15 \\
3\end{array}$ & 39,7 \\
\hline & $\begin{array}{l}\text { Employés/agent } \\
\text { s de maitrise }\end{array}$ & 158 & 41 & & $601-1200 \mathrm{fcfa}$ & $\begin{array}{c}13 \\
6\end{array}$ & 35,3 \\
\hline & Etudiant/élève & 36 & 9,3 & & $\begin{array}{l}1201-1800 \\
\text { fcfa }\end{array}$ & 14 & 3,6 \\
\hline & $\begin{array}{l}\text { Exploitant } \\
\text { agricole }\end{array}$ & 6 & 1,6 & & $>1800 \mathrm{fcfa}$ & 26 & 6,8 \\
\hline & Ouvriers & 122 & 31,7 & $\begin{array}{l}\text { Appréciation de } \\
\text { tchapalo }\end{array}$ & Goût & $\begin{array}{c}31 \\
8\end{array}$ & 82,6 \\
\hline & Retraite & 12 & 3,1 & consommable & Odeur & 19 & 4,9 \\
\hline & Sans emploi & 8 & 2,1 & & Aspect & 4 & 1,1 \\
\hline & Non répondant & 14 & 3,6 & & Autre & 10 & 2,6 \\
\hline \multirow{8}{*}{$\begin{array}{l}\text { Revenu } \\
\text { mensuel } \\
\text { (fcfa) }\end{array}$} & $<50000$ & 64 & 16,6 & & Non répondant & 34 & 8,8 \\
\hline & $\begin{array}{c}{[50000-100} \\
001[\end{array}$ & 147 & 38,2 & $\begin{array}{l}\text { Eléments de } \\
\text { différenciation }\end{array}$ & Degré d'alcool & $\begin{array}{c}20 \\
8\end{array}$ & 54 \\
\hline & $\begin{array}{c}{[100001-150} \\
001[\end{array}$ & 58 & 15,1 & $\begin{array}{c}\text { entre le tchapalo } \\
\text { du jour et celui }\end{array}$ & Goût & $\begin{array}{c}11 \\
8\end{array}$ & 30,6 \\
\hline & $\begin{array}{c}{[150001-200} \\
001[\end{array}$ & 28 & 7,3 & $\begin{array}{l}\text { de plus d'un } \\
\text { jour }(\%)\end{array}$ & Odeur & 21 & 5,5 \\
\hline & $\begin{array}{c}{[200001-250} \\
001[\end{array}$ & 12 & 3,1 & & Aspect & 3 & 0,8 \\
\hline & $\begin{array}{c}{[250001-300} \\
001[\end{array}$ & 6 & 1,5 & & Autre & 35 & 9,1 \\
\hline & $\geq 300001$ & 13 & 3,4 & Durée de & 1 jour & 29 & 7,5 \\
\hline & Non répondant & 57 & 14,8 & $\begin{array}{l}\text { conservation } \mathrm{du} \\
\text { tchapalo }(\%)\end{array}$ & 2 jours & $\begin{array}{c}10 \\
2\end{array}$ & 26,5 \\
\hline \multirow{2}{*}{$\begin{array}{l}\text { Volume } \\
\text { consommé } \\
\text { (litre) }\end{array}$} & $<1$ & 58 & 15,1 & & 3 jours & $\begin{array}{c}14 \\
3\end{array}$ & 37,1 \\
\hline & {$[1-2,1[$} & 177 & 46 & & 1 semaine & 89 & 23,1 \\
\hline
\end{tabular}




\begin{tabular}{cccccc}
{$[2,1-3,1[$} & 123 & 32 & 2 semaines & 1 & 0,3 \\
{$[3,1-4,1[$} & 16 & 4,1 & Plus & 21 & 5,5 \\
$>4,1$ & 11 & 2,8 & & & \\
\hline
\end{tabular}

\section{Consommation de tchapalo à Abidjan}

- Moyen de découvert du tchapalo et motivations premières à consommer le tchapalo

Le pourcentage des consommateurs ayant connu le tchapalo par le biais de leurs amis était élevé (Tableau 3). Des personnes interrogées, 40,6\% ont commencé à consommer le tchapalo sous l'influence de leurs groupes de paires, 26,2\% suite à des recommandations selon lesquelles le tchapalo aurait des vertus thérapeutiques qui concourt à prévenir le paludisme et favoriser la défécation. Environ $20 \%$ des consommateurs ont été motivé à consommer le tchapalo parce que le tchapalo est une boisson intégrée dans leur tradition et produite par leurs mères. Tous ces résultats indiquent que les Abidjanais dans leur majorité consomment le tchapalo par choix et non parce qu'elle fait partie de leur culture (Enou, 1997).

La principale raison de cette habitude de consommation était que le tchapalo permet de "se soigner" en l'occurrence, soigner le paludisme (Enou, 1997 ; Kouamé et al., 2015). Ainsi, quel que soit le groupe ethnique, la majorité des individus interrogés consommaient le tchapalo pour se soigner (54,3\%). Ensuite venaient ceux qui le consommaient pour d'autres raisons $(17,1 \%)$ telles que le tchapalo n'entraine aucun effet secondaire, il est nourrissant, moins cher et il permet d'oublier les problèmes. Ce résultat est conforme à celui de Louamy (2007) qui a montré que les Ivoiriens ont adopté le tchapalo pour ses vertus thérapeutiques.

\section{- Contexte social de consommation et mécanismes d'entretiens des liens sociaux}

Rares sont les personnes qui consommaient le tchapalo à domicile ; plus de $95 \%$ préfèrent le consommer au tchapalodrôme. La raison première évoquée était le plaisir de se retrouver entre amis $(26,8 \%)$, ensuite venaient ceux qui y consommaient juste pour le cadre $(22,1 \%)$ et pour l'ambiance $(17,9 \%)$ (Tableau 3). Ces résultats traduisent une certaine relation entre les consommateurs et le tchapalodrôme en ce sens que dans la société traditionnelle africaine, les repas sont souvent partagés en groupe entre les membres d'une même famille ou avec des connaissances.

Outre les relations entre eux, les consommateurs de tchapalo entretenaient également des liens de fraternités avec les productrices. Ainsi, $25,7 \%$ des consommateurs choisissaient de consommer dans un tchapalodrôme précis pour le goût de la boisson et 25,5\% le faisaient parce que la productrice leur réservait un bon accueil. Cela se perçoit à travers les 
« crédits » et les notions de « goûter voir » qui consiste à goûter la boisson avant de se décider à la consommer. Ces rapports sociaux orientent le consommateur dans le choix du tchapalodrôme.

\section{- Description des fréquences et des moments de consommation}

Les enquêtés qui consomment le tchapalo fréquemment étaient plus nombreux que ceux qui le faisaient occasionnellement. Parmi ceux qui consommaient fréquemment, il y a les consommateurs de la première catégorie $(58,7 \%)$ qui consommaient tous les jours et les consommateurs de la seconde catégorie $(36,6 \%)$ qui consommaient au moins une fois dans la semaine (Tableau 3). Ces résultats sont comparables à ceux de Akoma et al. (2013) qui ont rapporté que 68,2\% des consommateurs de kunun-zaki consomment cette boisson quotidiennement. Les moments favoris de consommation étaient l'après-midi $(42,9 \%)$ et la soirée $(19 \%)$. Ces heures choisies sont généralement des heures de descente du travail. Elles ont fini par rentrer dans les habitudes des consommateurs. En conséquence, même les week-ends, ces heures de consommation sont respectées.

Tableau 3 : Consommation de tchapalo à Abidjan

\begin{tabular}{|c|c|c|c|c|c|c|c|}
\hline \multirow[t]{2}{*}{ Observations } & \multirow[t]{2}{*}{$\begin{array}{l}\text { Caractéristi } \\
\text { ques socio- } \\
\text { culturelles }\end{array}$} & \multicolumn{2}{|c|}{$\begin{array}{l}\text { Consom } \\
\text { mateurs } \\
(\mathrm{n}=385)\end{array}$} & \multirow[t]{2}{*}{ Observations } & \multirow[t]{2}{*}{$\begin{array}{l}\text { Caractéristi } \\
\text { ques socio- } \\
\text { culturelles }\end{array}$} & \multicolumn{2}{|c|}{$\begin{array}{l}\text { Consom } \\
\text { mateurs } \\
(n=385)\end{array}$} \\
\hline & & $\begin{array}{l}\text { Eff } \\
\text { ecti } \\
\text { fs }\end{array}$ & $\begin{array}{l}\text { Pour } \\
\text { cent } \\
\text { age } \\
(\%)\end{array}$ & & & $\begin{array}{l}\text { Eff } \\
\text { ecti } \\
\text { fs }\end{array}$ & $\begin{array}{l}\text { Pour } \\
\text { cent } \\
\text { age } \\
(\%)\end{array}$ \\
\hline \multirow{4}{*}{$\begin{array}{c}\text { Moyen de } \\
\text { découverte du } \\
\text { tchapalo }(\%)\end{array}$} & $\begin{array}{l}\text { Par ma } \\
\text { femme }\end{array}$ & 5 & 1,3 & \multirow{6}{*}{$\begin{array}{c}\text { Raison de } \\
\text { consommation au } \\
\text { tchapalodrôme }\end{array}$} & Ambiance & 69 & 17,9 \\
\hline & $\begin{array}{l}\text { Par mes } \\
\text { amis }\end{array}$ & $\begin{array}{c}16 \\
7\end{array}$ & 43,4 & & $\begin{array}{c}\text { Convivialit } \\
\text { é }\end{array}$ & 20 & 5,2 \\
\hline & $\begin{array}{l}\text { Produit de } \\
\text { mon terroir }\end{array}$ & 90 & 23,4 & & Le cadre & 85 & 22,1 \\
\hline & Autre & $\begin{array}{c}12 \\
3\end{array}$ & 31,9 & & $\begin{array}{l}\text { Retrouver } \\
\text { ses amis }\end{array}$ & $\begin{array}{c}10 \\
3\end{array}$ & 26,8 \\
\hline $\begin{array}{l}\text { Motivations } \\
\text { premières à }\end{array}$ & Curiosité & 51 & 13,2 & & $\begin{array}{c}\text { Se faire des } \\
\text { amis }\end{array}$ & 29 & 7,5 \\
\hline \multirow[t]{3}{*}{$\begin{array}{c}\text { consommer le } \\
\text { tchapalo }\end{array}$} & $\begin{array}{l}\text { Effet de } \\
\text { groupe }\end{array}$ & $\begin{array}{c}15 \\
6\end{array}$ & 40,6 & & Autre & 79 & 20,5 \\
\hline & $\begin{array}{l}\text { Recomman } \\
\text { dation }\end{array}$ & $\begin{array}{c}10 \\
1\end{array}$ & 26,2 & \multirow{4}{*}{$\begin{array}{c}\text { Raisons de } \\
\text { préférence d'un } \\
\text { tchapalodrôme à } \\
\text { un autre }\end{array}$} & Amis & 56 & 14,5 \\
\hline & Autre & 77 & 20 & & $\begin{array}{c}\text { Bon accueil } \\
\text { de la } \\
\text { clientèle }\end{array}$ & 98 & 25,5 \\
\hline $\begin{array}{c}\text { Raisons } \\
\text { d'habitude de }\end{array}$ & $\begin{array}{l}\text { Aucune } \\
\text { raison }\end{array}$ & 26 & 6,8 & & Goût & 99 & 25,7 \\
\hline consommation & $\begin{array}{c}\text { Boisson } \\
\text { locale }\end{array}$ & 19 & 4,9 & & $\begin{array}{l}\text { A proximité } \\
\text { du lieu } \\
\text { d'habitation }\end{array}$ & 88 & 22,9 \\
\hline
\end{tabular}




\begin{tabular}{|c|c|c|c|c|c|c|c|}
\hline & $\begin{array}{l}\text { Faire des } \\
\text { rituels }\end{array}$ & 15 & 3,9 & & Autre & 44 & 11,4 \\
\hline & $\begin{array}{l}\text { Plaisir de } \\
\text { boire }\end{array}$ & 30 & 7,8 & \multirow[t]{4}{*}{$\begin{array}{l}\text { Fréquence de } \\
\text { consommation }\end{array}$} & $\begin{array}{l}\text { Tous les } \\
\text { jours }\end{array}$ & $\begin{array}{c}22 \\
6\end{array}$ & 58,7 \\
\hline & Se distraire & 8 & 2,1 & & $\begin{array}{l}\text { Une fois } \\
\text { par semaine }\end{array}$ & $\begin{array}{c}14 \\
1\end{array}$ & 36,6 \\
\hline & $\begin{array}{l}\mathrm{Se} \\
\text { retrouver } \\
\text { entre amis }\end{array}$ & 12 & 3,1 & & $\begin{array}{l}\text { Une fois } \\
\text { chaque } \\
\text { deux } \\
\text { semaines }\end{array}$ & 8 & 2,1 \\
\hline & Se soigner & $\begin{array}{c}20 \\
9\end{array}$ & 54,3 & & $\begin{array}{l}\text { Une fois } \\
\text { par mois }\end{array}$ & 10 & 2,6 \\
\hline \multirow{5}{*}{$\begin{array}{l}\text { Préférence de } \\
\text { lieu de } \\
\text { consommation }\end{array}$} & Autre & 66 & 17,1 & \multirow{5}{*}{$\begin{array}{l}\text { Moment de } \\
\text { consommation }\end{array}$} & Matin & 57 & 14,8 \\
\hline & $\begin{array}{c}\mathrm{Au} \\
\text { tchapalodrô } \\
\text { me }\end{array}$ & $\begin{array}{c}36 \\
8\end{array}$ & 95,6 & & Midi & 25 & 6,5 \\
\hline & A domicile & 15 & 3,9 & & Après-midi & $\begin{array}{c}16 \\
5\end{array}$ & 42,9 \\
\hline & \multirow[t]{2}{*}{ Autre } & 2 & 0,5 & & Soirée & 73 & 19 \\
\hline & & & & & Toute heure & 65 & 16,8 \\
\hline
\end{tabular}

\section{Conclusion}

Il ressort de cette enquête que le tchapalo, bière traditionnelle ivoirienne, originaire du Nord de la Côte d'Ivoire, est produite exclusivement par les femmes Burkinabés et les femmes du nord de la Côte d'Ivoire, en l'occurrence, les femmes de l'ethnie lobi. Elles sont le plus souvent illettrées et ont pour activité la production et commercialisation du tchapalo à Abidjan. Cette activité est une source de revenue non négligeable leur permettant de prendre en charge leurs familles. Le tchapalo est consommé par toutes les couches socio-économiques et professionnelles sans distinction de nationalité. La majorité des consommateurs ivoiriens appartient au groupe ethnique Akan. Toutes les couches socio-économiques consomment le tchapalo. Les ouvriers et les agents de maîtrise sont les plus représentés. Toutefois, les personnes à faible revenu sont les plus nombreux. Parmi les enquêtés, se trouvent certains qui consomment le tchapalo pour la première fois par le biais de leurs amis ; tandis que, d'autres le consomment sur recommandation et y restent attachés. Les habitués trouvent des vertus thérapeutiques au tchapalo telles que la prévention du paludisme et la facilitation de la défécation. De plus, la consommation du tchapalo à Abidjan est caractérisée par un ensemble de facteurs s'inscrivant dans un champ social spécifique qu'est le tchapalodrôme et est liée à des logiques économiques. D'autres logiques sociales telles que l'échange sans monnaie existent entre les consommateurs et les productrices. De ce fait, le choix des tchapalodrômes est déterminé par les affinités qui lient les consommateurs entre eux et aux brasseuses. Les après-midis et les soirées sont les heures privilégiées pour la consommation du tchapalo. 


\section{Remerciements}

Ce projet de recherche a été financé par le Programme d'Appui Stratégique à la Recherche Scientifique (PASRES). Nous exprimons nos remerciements au comité de Pilotage du PASRES pour son appui financier. Nos remerciements vont également à l'endroit des productrices de tchapalo, des consommateurs et de tous les acteurs ayant participé aux différentes enquêtes.

\section{References:}

1. Abdoul-latif F. M., Bayili R. G., Obame L.-C., Bassolé I. H. N. \& Dicko M. H. (2012). Comparison of phenolic compounds and antioxidant capacities of traditional sorghum beers with other alcoholic beverages. African Journal of Biotechnology, 11(81) : 14671- 14678.

2. Abdoul-latif F. M., Bassolé I. H. N. \& Dicko M. H. (2013). Proximate composition of traditional local sorghum beer dolo manufactured in Ouagadougou. African Journal of Biotechnology 12(13) : 1517-1522.

3. Aka S., Camara F., Nanga Y. Z., Loukou Y. G. \& Dje K. M. (2008). Evaluation of organic acids and sugars contents during the production of « tchapalo », a traditional sorghum beer in Côte d'Ivoire. Journal of Food Technology, 6(5): 189-195.

4. Aka S., N'Guessan K. F., Nanga Y. Z., Loukou Y. G., Mazabraud A. I. \& Djè K. M. (2010). Characterization of Lactobacillus species isolated from mash, sour wort and tchapalo produced in Côte d'Ivoire. Food 4(1): 49-54.

5. Akoa E. E. F., Kra K. A. S., Megnanou R.-M., Akpa E. E. \& Ahonzo N. L. S. (2012). Sensorial characteristics of a senescent plantain empiric dish (Dockounou) produced in Côte d'Ivoire. Journal of Food Research, 1 (4) : 150-159.

6. Akoma O., Agarry O. O. \& Nkama I. (2013). A Study on the production and consumption pattern of kunun-zaki: a cereal based ethnic fermented beverage of northern Nigeria. British Journal of Applied Science \& Technology 3(4): 1220-1227, 2013.

7. Amané N. D, Assidjo N. E, Gbongue M. A, Bohoussou K. \& Cardot P. (2005). Caractérisation physico-chimique d'une bière traditionnelle ouest Africaine : le tchapalo. Agronomie Africaine, 17(2): 143-152.

8. Amané D. N., Kouamé K. B., Kouamé C. \& Assidjo E. N. (2012). Optimisation du procédé de fabrication du «tchapalo », bière traditionnelle ivoirienne par le plan factoriel fractionné. Afrique Science, 8(3) : 69-81.

9. Djanan D., Mbayhoudel K. \& Nanadoum M. (2002). Organisation des unités de transformation artisanale en zone de savanes : Cas de la 
transformation du sorgho en bière locale bili-bili à Moundou au Tchad. In J.Y. Jamin \& L. S. Boucar (Ed). Savanes africaines : des espaces en mutation des acteurs face à de nouveaux défis. Cirad-Prasac, actes du colloque, Garoua, Cameroun.

10. Djè M. K., N'Guessan K. F., Djeni T. N. \& Dadié T. A. (2008). Biochemical changes during alcoholic fermentation in the production of tchapalo, a traditional sorghum beer. International Journal of Food Engineering, 4(7) art. 2.

11. Enou A. A. W. (1997). La bière de sorgho ou tchapalo : analyses, fabrication et consommation, approche analytique. Thèse de pharmacie de la faculté de pharmacie, université de Cocody, Abidjan, $110 \mathrm{p}$.

12. INS (Institut national de la statistique de Côte d'Ivoire), (2015). Recensement Général de la Population et de l'Habitat 2014 de Côte d'Ivoire. Conseil des ministres de rentrée gouvernementale Mercredi 03 septembre 2014. Abidjan-Palais de la Présidence de la République. http://news.abidjan.net/h/518921.html

13. INS/ICF, (2012). Enquête Démographique et de Santé et à Indicateurs Multiples de Côte d'Ivoire 2011-2012. Calverton, Maryland, USA: INS et ICF International, $561 \mathrm{p}$.

14. Kayodé A. P. P. (2006). Diversity, users' perception and food processing of sorghum: implications for dietary iron and zinc supply. Ph.D. thesis Wageningen university, the Netherlands.

15. Kouame K. B., Koko A. C., Masse D. \& Assidjo N. E. (2015). Batch fermentation process of sorghum wort modeling by artificial neural network. European Scientific Journal, 11(3) : 75-93,

16. Louamy J. (2007), Tchapalo : une boisson thérapeutique, rézoivoire.net. En ligne, consulté le 10-08-2015, http://rezoivoire.net;

17. Magrin G. \& Mbayhoudel K. (2005). La bière à l'index ? Enjeux et dynamiques de la consommation d'alcool au sud du Tchad, In C. Raimond (Ed). Ressources vivrières et choix alimentaires dans le bassin $d u$ lac Tchad, Prodig, pp. 501-526, Paris. http://www.documentation.ird.fr;

18. Maoura N., Mbaiguinam M., Gaillardin C. \& Pourquie J. (2006). Suivi technique, analytique et microbiologique de la «bili bili », bière traditionnelle tchadienne. Afrique Science, 20(1) : 69-82.

19. N'Guessan K. F. (2009). Analyse de quelques paramètres de la fermentation alcoolique conduisant au tchapalo (bière traditionnelle à base de sorgho) et des potentialités à la formulation d'une culture starter à partir des levures isolées. Thèse de Doctorat, Université d'Abobo-Adjamé, Abidjan. 
20. Oyewole O. A. \& Isah P. (2012). Locally fermented foods in Nigeria and their significance to national economy: a Review. Journal of Recent Advances in Agriculture, 1(4) : 92-102.

21. Pale S., Sibiri J.-B. T., Bougouma B. \& Maso S. C. (2010). Sorghum malt and traditional beer (dolo) quality assessment in Burkina Faso. Ecology of Food and Nutrition, 49 : 129-141.

22. Pale S., Kabore D. P., Taonda S. J. B., Bougouma B. \& Mason S. C. (2011). Economic assessment of malt and traditional beer (dolo) production in Burkina Faso. Journal of Development and Agricultural Economics, 3(15) : 689-694.

23. Yaméogo G., Yélémou B., Kaboré O. \& Traoré D. (2013). Bois d'énergie du 'dolo' et bois de service : deux modes de consommation de bois à Vipalogo au Burkina Faso. Bulletin de la Recherche Agronomique du Bénin, 73 : 1-12.

24. Yao A. K., Kadio N., Coulibaly A. \& Agbo G. N. (1995). Production du tchapalo à partir du sorgho en Côte d'Ivoire. In J.M. Menyonga, T. Bezuneh, C.C. Nwasike, P.M. Sedogo, A. Tenkouano (Ed). Processing and industrial utilization of sorghum and related cereal in Africa. Proceeding of the OUA/STRC-SAFGRAD regional symposium 22-26 Nov 1993 pp. 55-60, Ouagadougou, Burkina Faso.

25. Zongo M. (2003). La diaspora Burkinabé en Côte d'Ivoire : trajectoire historique, recomposition des dynamiques migratoires et rapport avec le pays d'origine. Revue africaine de sociologie, 7 (2) : 58-72. 